\title{
Thin layer fluorescence microscopy based on one-dimensional photonic crystal
}

\author{
Kirill Prusakov ${ }^{1,2, *}$, Dmitry Basmanov ${ }^{1}$, and Dmitry Klinov ${ }^{1}$ \\ ${ }^{1}$ Federal State Budgetary Institution Federal Research and Clinical Center Of Physical-Chemical \\ Medicine, Federal Medical Biological Agency, Laboratory of Medical Nanotechnologies, 119435 \\ Malaya Pirogovskaya 1a, Moscow, Russia \\ ${ }^{2}$ Moscow institute of physics and technology, Departure of Biological and Medical Physics, 141701 \\ Institutskiy per. 9, Dolgoprudny, Moscow Region, Russia
}

\begin{abstract}
A new method of specimen illumination for wide-field fluorescence microscopy has been presented. This method allows to excite the fluorescence in a thin near-surface layer of the studied object. As a result, the captured images have greater contrast and signal-to-background ratio in comparison with the epifluorescence ones. The long-range surface waves in one-dimensional photonic crystal have been used to localize the electromagnetic field exciting the fluorescence. An experimental setup has been created to excite the surface waves and obtain images of the objects from the near-surface layer. For an illustration of the possibilities of our method, we conducted several experiments with specimens that are typical for fluorescence microscopy, such as bacteria and eukaryotic cells.
\end{abstract}

\section{Introduction}

One-dimensional photonic crystal (1D PC) is a multilayer meta-structure with periodically changing refractive indices of the layers. As shown by V. Konopsky et al. [1], it is possible to create the special conditions for existence of one or several modes of longrange surface optical range electromagnetic waves on the surface of 1D PC. The main idea of our work [2] is to use a small penetration depth of the long-range surface waves to improve the contrast and signal-to-background ratio of the images that could be captured with the help of a wide-field fluorescence microscope. One of the most frequently used methods of such kind image improvement is the so-called Total Internal Reflection Fluorescence Microscope (TIRFM). However usually, this approach requires permanent fine-tuning of optical setup and works only with high numeric aperture lenses. It will be shown that our method does not have such limitations.

\section{Materials and Methods}

The 1D PC structure was calculated using the method of complex impedance [3]. The dispersion characteristic of the calculated structure corresponded to the excitation spectra of

\footnotetext{
*Corresponding author: kaprusakov@gmail.com
} 
green fluorescent protein (GFP). This structure provides an opportunity to initiate longrange surface waves with $473 \mathrm{~nm}$ wavelength on the 1D PC surface. The PC was fabricated using the magnetron sputtering and consisted of 3 layers of $\mathrm{SiO}_{2}$ and $\mathrm{Ta}_{2} \mathrm{O}_{5}(134.4 \mathrm{~nm}$ and $78.4 \mathrm{~nm}$ thickness respectively) and a final layer of $\mathrm{SiO}_{2} 300 \mathrm{~nm}$ thick. In order to excite the long-range surface waves, an experimental setup based on the Kretschmann configuration with a cylindrical lens was created (see Fig. 1). This configuration does not require permanent fine-tuning of the laser illumination angle.

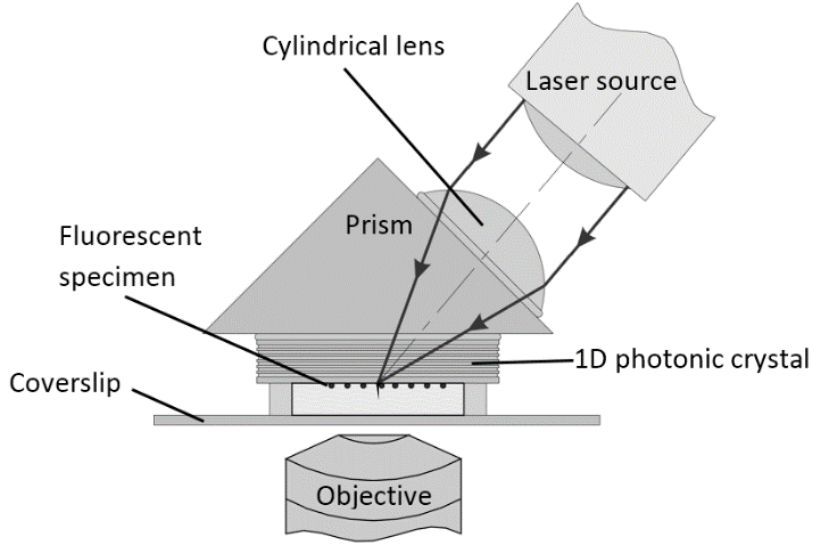

Fig. 1. An experimental setup based on the Kretschmann configuration.

Laboratory mutant strain of GFP labeled E. coli and a mutant line of GFP labeled HeLa cells were used as the representative specimens for fluorescent microscopy. Cells were fixed on the PC surface. The images of the PC surface were captured with the help of a wide-field fluorescence microscope.

\section{Results and Conclusion}

The experiments showed that fluorescence is excited only inside a thin near-surface layer $(\sim 150 \mathrm{~nm})$ of a specimen. Moreover, in the obtained images the signal-to-background ratio is on the average 7 times greater than the value of this parameter in the epifluorescent images of the same specimens. Thus, the localization of the initiating radiation reduces a background signal from the specimen bulk. As a result, it became possible to study a more detailed structure of a specimen. This new method of specimen illumination can be used as a cheap and simple alternative to the TIRFM method. Also, it potentially can be combined with a surface waves biosensor [4].

This work was supported by the Russian science foundation (project № 17-75-30064).

\section{References}

1. V.N. Konopsky, E.V. Alieva, Phys. Rev. Lett., 97, 253904 (2006)

2. K.A. Prusakov, D.V. Basmanov, D.V. Klinov, Patent RU 2626269 (2017)

3. V.N. Konopsky, New J. Phys., 12, 093006 (2010)

4. M. Prokofjeva, V. Tsvetkov, D. Basmanov, A. Varizhuk, M. Lagarkova, I. Smirnov, K. Prusakov, D. Klinov, V. Prassolov, G. Pozmogova, S. N. Mikhailov, Nucleic Acid Ther., 27, 56 (2017) 